
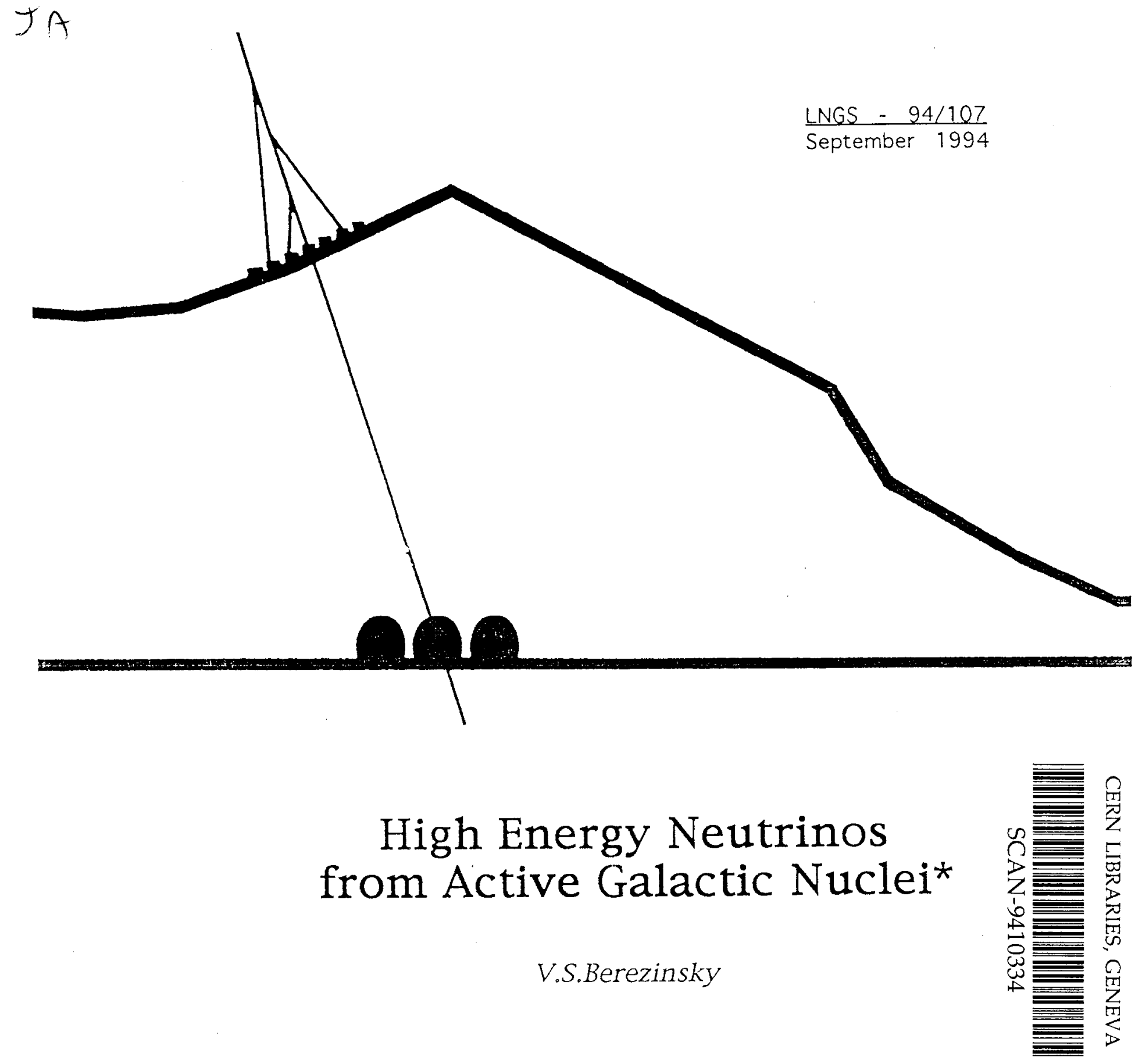

*invited talk given at "NEUTRINO-94", Eilat, Israel, May29-June3, 1994 


\title{
High Energy Neutrinos from Active Galactic Nuclei
}

\author{
V.Berezinsky \\ INFN, Laboratori Nazionali del Gran Sasso, 67010 Assergi (AQ), Italy \\ and \\ Institute for Nuclear Research, Moscow, Russia
}

The neutrino production in $A G N$ is reviewed with the emphasis on the diffuse neutrino flux. Most attention is given to the AGN models with the shock acceleration. The maximum energy of acclerated protons and thus of neutrinos is analysed. The maximum energy is shown to increase with the mass of black hole $M$ and to decrease as neutrino luminosity $L_{\nu}$ increases. Neutrinos with the hirhest energies in the diffuse spectrum are produced by the present-day sources with small $L_{\nu}$ and large $M$, while the low-energy part of the spectrum is produced by evolving sources (with large $L_{\nu}$ ) at large redshifts. The analysis presented here suggests that maximum neutrino energy in the diffuse spectrum is limited by $10^{3}-10^{6} \mathrm{GeV}$ and therefore the detectable diffuse flux looks like a (significant) bump over atmosheric neutrino spectrum.

\section{Introduction}

Apart from the point-like sources high energy neutrino astronomy is connected with the diffuse flux. Its detection is possible only at very high energies where atmospheric neutrino flux becomes small. The anticipated sources of diffuse neutrino flux are $A G N$, bright phase in the galaxy evolution [1-3] and decays of the massive relic particles [4-5] including neutralino [6].

There are several models for $A G N$ as neutrino sources. All of them are developed as the hidden sources, for which accompaning gamma radiation is suppressed. Without this condition the neutrino flux is strongly limited by the observed gamma ray flux.

In this paper the AGN models for neutrino production are discussed. The early works [7] include the calculations of neutrino fluxes from quasars with their evolution taken into account and the Seyfert galaxies [8] (without assumption of the evolution). The $p \gamma$ collisions inside AGN were considered as the main mechanism of neutrino production [9].

The recent calculations for neutrino production in AGN include as the basic element the acceleration of protons by the shock in the accreting gas. These models predict considerably higher fluxes

"Invited talk given at "NEUTRINO-94", Eilat, Israel, May29 - June 3,1994 at lower energies. For the recent comprehensive review see ref. $[10 j$.

2. Neutrino fluxes in the AGN model with the shock acceleration

The essence of this model can be described as follows.

The accretion of the gas onto a massive black hole provides the energy supply. It is tacitly assumed in the calculations that the accretion is quasi-spherical, though the authors usually speak about radiation from the disk. One can consider a thick accretion disk with the width of the order of the radial distance to reconcile both ideas. The radial flow of accreting gas is assumed to be terminated by a shock at radial distance $r_{s h}=20 r_{g}$, where $r_{g}=G M / c^{2}=1.5 \cdot 10^{13} M_{8} \mathrm{~cm}$, and $M=M_{8} \cdot 10^{8} M_{\odot}$ is the mass of the black hole. The protons are accelerated by the Fermi I mechanism at the shock and produce neutrinos in $p \gamma$ collisions with the ambient photons (see Fig.1). The flux of these photons and their spectrum are taken from the observations (the continuum radiation data). According to the observations, these photons have a power-law spectrum with index $\alpha=-1.7$ and with UV bump centered at $\epsilon \sim 30 \mathrm{eV}$. A remarkable feature of this model is that only high energy neutrinos and X-rays (including soft gamma- radiation with energies less 
than $1 \mathrm{MeV}$ ) can escape from the source. The protons accelerated at the shock cannot diffuse upstream, rather they are dragged by the gas flow downstream until they are trapped by the black hole. The $\mathrm{X}$ - and gamma- rars are absorbed due to $\gamma+\gamma \rightarrow e^{+}+e^{-}$- collisions. In the model [11] even high energy neutrons are absorbed in $n \gamma$ collisions.

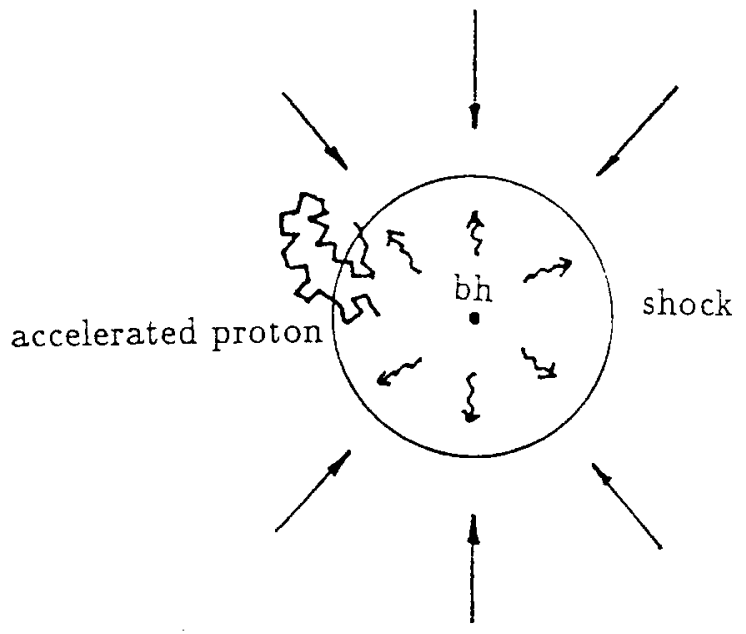

Fig.1. AGN model with shock acceleration. Wavy arrows symbolize photons of the continuum radiation (optical and X-ray radiation). A trajectory of an accelerated proton is shown by the broken line.

The model described above has several open problems.

First of all, the existence of the standing shock is just an assumption. Moreover, for the effective neutrino production the shock should be at the distance $10 r_{g} \leq r_{s h} \leq 30 r_{g}$. At smaller distances the maximum energy of accelerated protons becomes small, for larger distances the neutrino flux diminishes.

It is not demonstrated that the spectrum of the target photons is reproduced due to $p \gamma$ collisions with the observed shape $\left(n_{x} \propto E_{x}^{-1.7}\right)$. In other words, the self-consistency of the model still remains an open problem.

Finally, as demonstrated in ref's[12-13] there is an instability which results in exponential growth of number of photons produced by electronpositron pairs. This instability can diminish the maximum energy of accelerated protons down to $E_{c} \sim 1 \mathrm{TeV}^{-}$.

However, for the model outlined above with two basic assumptions (existence of the shock at the distance $r_{s h} \sim 20 r_{g}$ and the production of continuum radiation with spectrum $n_{x} \propto E^{-1.7}$ ) the neutrino flux can be calculated rather reliably.

A compilation of the recent calculations for $\nu_{\mu}+\bar{\nu}_{\mu}$ the diffuse neutrino fluxes from $A G N$ is given in Fig.2. The reference $f$ lux is that of atmospheric neutrinos (atm): only fluxes above it are detectable.

The pioneering calculations of this kind were made in refi11] (see refi 14 ] and erratum [15] for reconsideration of neutrino flux). Only py collisions were taken into account in this work. The flux is given in Fig.2 by the curve SDSS.

The most detailed calculations were performed in ref's[16-17] for the model basically identical with that of refi11?. In computer simulation a proton with the low initial energy $E_{0}$ is injected in the acceleration region and then is followed with rate of acceleration, rate of energy losses and probability of diffusive escape taken into account. The maximum energy is determined by equality of rate of acceleration and rate of energy losses. The magnetic field is taken from equipartition equilibrium with the radiation field. Note, that the authors did not use the analytical expression for the spectrum of accelerated particles, but just included the process of acceleration in the Monte-Carlo simulation together with energy losses. The calculated spectra are shown in Fig.2 by the curves $\mathrm{SP}(\mathrm{a})$ and $\mathrm{SP}(\mathrm{b})$ for two different assumptions about radial velocity of the gas flow. One can notice the dramatic increase of the neutrino flux at energy $E<10^{5} \mathrm{GeV}$ in comparison with SDSS curve. It is the consequence of $p p$ neutrino production taken into account.

Somewhat different model is used in calculations of ref[18]. The disk accretion model is assumed there. The particles are accelerated by the shock located nearby the inner radius of the accretion disk. The accelerated protons produce neutrinos due to pp-collisions in the disk. Nontransparency for hard $X$-rays and soft $\gamma$ radiation 
is not explicitly shown in the paper. The maximum neutrino energy is given by

$E_{\nu}^{\max }=3.5 \cdot 10^{5} \frac{u_{s h}}{c}\left(a B_{4}\right)^{-1 / 2} \mathrm{GeV}$,

where $u_{s h}$ is the shock speed (we take for it the maximum value $c / \sqrt{3}$ allowed in this model), $a$ is the ratio of radiation energy density to magnetic energy density (for $B=1 \cdot 10^{3} G$ and $L_{\nu}=1$. $10^{45} \mathrm{erg} / \mathrm{s}$ we estimate it to be 1.7$)$ and $B_{\text {t }}$ is the magnetic field in units $10^{-4} \mathrm{G}$. In this case the maximum neutrino energy is $5 \cdot 10^{\overline{5}} \mathrm{GeV}$ as shown in Fig. 2 by curve Bi.

The calculations of ref[19] are performed in more self-consistent fashion, from the point of view of production of the continuum radiation. The calculated flux for redshift $z=5$ is shown in Fig. 2 by the curve SB.

The highest neutrino energy can be obtained in the model suggested in ref $[20]$, where protons are accelerated in the jet far away from the black hole.

In Fig. 2 are also shown the flux from the early calculations [7] (curve $\mathrm{Be}$ ) and the flux from the bright phase in the galaxy evolution $[1]$ (the shaded area).

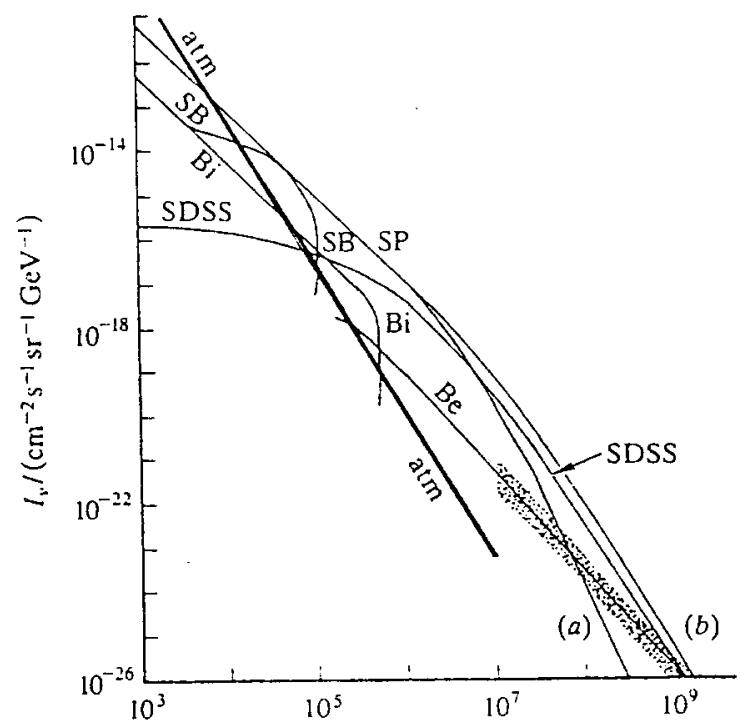

Fig.2. Diffuse fluxes of $\nu_{\mu}+\bar{\nu}_{\mu}$ neutrinos from AGN. The following notation is used: atm, atmospheric neutrino flux; SDSS, ref [21]; $\mathrm{SP},(\mathrm{a})$ and (b), ref[16]; $\mathrm{SB}$, ref [19]; $\mathrm{Bi}$, ref [18], and $\mathrm{Be}$, ref[ $[7]$. The shaded area refers to the bright phase in galaxy evolution [1].

As one can see from Fig.2 the crucial problem for detectability of diffuse neutrino flux is the maximum energy for the accelerated protons and thus for neutrinos.

We shall consider here this problem in some detail.

\section{Maximum neutrino energy}

Let us consider first the energy production due to the accretion. The total energy release for the spherical accretion is about $10 \%$ of gravitational energy release at the Schwartzschild radius, $2 r_{g}$. To maximize this energy we assume that it is radiated away in the form of X-rays. Then the X-ray luminosity is

$$
L_{x} \approx 0.1 G M M / 2 r_{g}
$$

where $G$ is the gravitational constant, $M$ is the mass of the black hole and $\dot{M}$ is the rate of the accretion.

The acceleration is powered by gravitational energy release at $r=r_{s h}$. Let us assume that $\sim 10 \%$ of this energy is transferred to accelerated protons. Then for the proton luminosity we have

$L_{p} \approx 0.1 G M \dot{M} / r_{s h}$,

For the thick photon target the neutrino luminosity is

$L_{\nu} \approx 0.5 L_{p}$.

From (2) -(4) it follows

$L_{x} / L_{\nu} \approx r_{s h} / r_{g} \approx 20$.

Let us assume further that this X-ray radiation is reprocessed by the cascade process to the powerlaw spectrum

$n_{x}(\epsilon) \propto \epsilon^{-1.7}$,

with a cut off at $\epsilon_{m} \approx 1 \mathrm{MeV}$. Then we obtain for the number density of the photons which are 
able to photodissociate a proton with energy $E_{p}$ to a nucleon and pion:

$n_{x}(>\epsilon)=\frac{60}{7 \pi} \frac{L_{\nu}}{c r_{s h}^{2} \epsilon_{m}}\left(\frac{\mu m_{p}}{E_{p} \epsilon_{m}}\right)^{-0 . i}$,

where $\mu$ and $m_{p}$ are the masses of pion and proton, respectively.

The maximum proton energy $E_{p}^{\text {max }}$ can be found equalizing the acceleration time

$t_{a c c}=\frac{3}{u_{1}-u_{2}}\left(\frac{D_{1}}{u_{1}}+\frac{D_{2}}{u_{2}}\right)$

and the characteristic time of energy losses due to photopion production

$t_{\text {loss }}=\left(0.5 \sigma_{p-n_{x}}(>\epsilon) c\right)^{-1}$.

In (8) and (9) $u_{1}$ and $u_{2}$ are the fluid velocities upstream and downstream, respectively $\left(u_{1} / u_{2}=4\right.$ for strong shock), $D_{1}$ and $D_{2}$ are the diffusion coefficients upstream and downstream, respectively, $\sigma_{p}$ is the cross section of pion production, and $n_{x}(>\epsilon)$ is given by $(7)$. Using for the diffusion coefficients $D \approx r_{B} c$, where $r_{B}=E / e B$ is the giroradius and $B$ the magnetic field, we obtain

$\frac{E_{p}^{m a x}}{\sqrt{\mu m_{p}}}=\left(\frac{7 \pi}{600} \frac{e B c r_{s h}^{2}}{L_{\nu} \sigma_{p \gamma}} \frac{u_{1}^{2}}{c^{2}}\right)^{0.588}\left(\frac{\epsilon_{m}}{\sqrt{\mu m_{p}}}\right)^{0.17}$

Putting in (10) numerical values $B=1 \cdot 10^{3} \mathrm{G}$, $\epsilon_{m} \approx 1 \mathrm{MeV}, \sigma_{p \gamma}=1 \cdot 10^{-28} \mathrm{~cm}^{2}$ and $u_{1}=\xi u_{f f}$, where $u_{f j}=c\left(2 r_{g} / r_{s h}\right)^{1 / 2}$ is the free-fall velocity at the location of the shock $r_{s h}=20 r_{g}$, and $\xi$ is a numerical coefficient with the value between $1 / 3$ and $1 / 10$, we obtain (for $\xi=0.1$ )

$E_{p}^{\max }=9.3 \cdot 10^{5} L_{45}^{-0.588} M_{8}^{1.18} \mathrm{GeV}$

where $L_{45}$ is the neutrino luminosity in units of $10^{45} \mathrm{erg} / \mathrm{s}$. The related cut-off energy in the diffuse neutrino spectrum is

$E_{\nu}^{\max } \approx 1.6 \cdot 10^{4} L_{4 \overline{5}}^{-0.588} M_{8}^{1.18}(3 /(1+z) \mathrm{GeV}$.

where $z$ is redshift of the cosmological epoch with the most effective neutrino production. If an ultraviolet bump is also formed at $r \sim 2 r_{g}$, then it is enough to have $L_{C r}=0.01 G M \dot{M} /\left(2 r_{g}\right)$ for the effective energy losses of protons. The maximum energy of the proton is determined in this case by kinematical condition of pion production on photons with energy $\epsilon_{L \uparrow} \approx 30 \mathrm{eV}$ :

$E_{p}^{\operatorname{mar}} \sim \mu m_{p} / \epsilon_{C \cdot} \sim 4 \cdot 10^{6} \mathrm{GET}$

which is almost the same as given by (11).

As one can see, these calculations coincide well with the lowest $E_{p}^{\text {max }}$ estimate from ref.[19].

\section{Discussion}

It is instructive to compare our calculations for $E_{p}^{\max }$ with the previous calculations. We choose for comparison the detailed calculations of ref[1617]. In Fig. 3 we plotted the proton energy losses divided by the energy density of the continum radiation at the location of the shock; as it is presented in ref $[16]$.

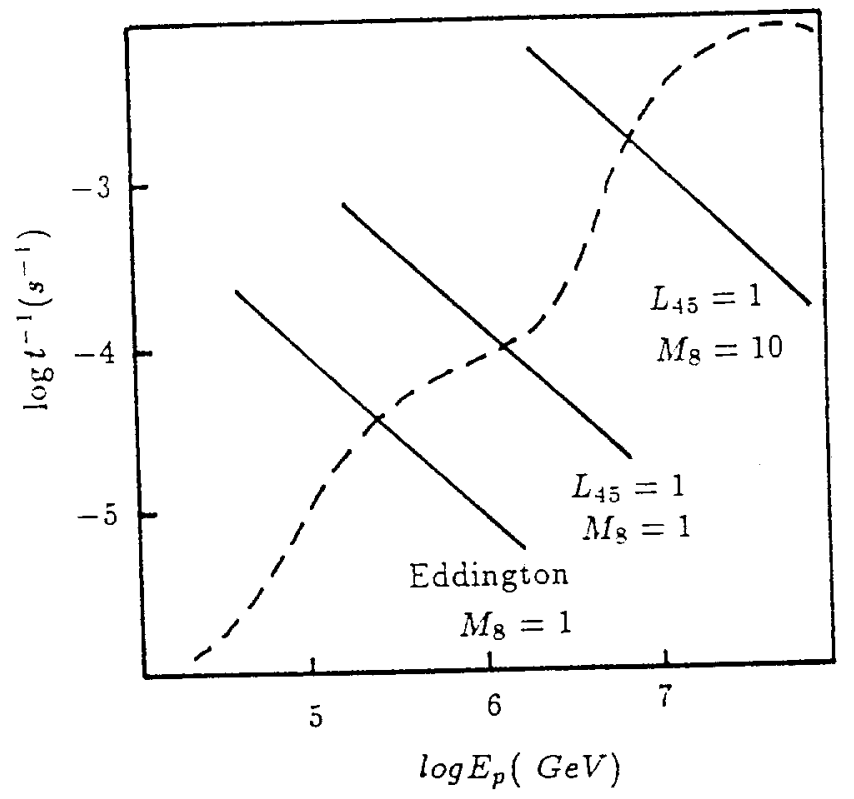

Fig.3. Maximum energy of accelerated proton. Dashed curve gives $t_{\text {loss }}^{-1}=$ $u_{r a d}^{-1} E_{p}^{-1} d E_{p} / d t$ according to calculations of ref[16] (for definition of $u_{\text {rad }}$ see the text). The straight lines give $u_{\text {rad }}^{-1} t_{a c c}^{-1}$ as function of proton energy. The curve Eddington corresponds to the Eddington regime for $M_{8}=1$. For the numerical values of the parameters see the text. The points of intersection $\left(t_{\text {loss }}=t_{a c c}\right.$ ) give the maximum energy of accelerated protons. 
More precisely we plotted in Fig.3. the values

$t_{\text {loss }}^{-1}=u_{\text {rad }}^{-1} E_{p}^{-1} d E_{p} / d t$,

where

$u_{\text {rad }}=U_{\bar{G}}^{-1} L_{\text {cont }} / \pi r_{s h}^{2} c=L_{45} / x_{10}^{2} M_{8}^{2}$,

$U_{0}=7.57 \cdot 10^{16} \mathrm{eV} / \mathrm{cm}^{3}, L_{45}=L_{c o n t} / 10^{45} \mathrm{erg} / \mathrm{s}$ and $x_{10}=r_{s h} / 20 r_{g}$. The straight lines show the acceleration time calculated according to Eq.(8)and assuming that $D_{1}=D_{2}=\bar{D} r_{B} c$ and $u_{1}=\xi u_{f f}$, where $u_{f f}=c\left(2 r_{g} / r_{s}\right)^{1 / 2}$ is the freefall velocity at the location of the shock. In fact, the plotited values are $u_{\text {rad }}^{-1} t_{a c c}^{-1}$. The curves in the graph are given for the values of the parameters $B=1 \cdot 10^{3} G, x_{10}=1, \xi=0.1$, and $\bar{D}=3.3$. The points of intersection $\left(t_{\text {loss }}=t_{\text {acc }}\right)$ give the maximum energy of accelerated protons.

We see that these calculations are in very reasonable agreement with our analytical estimates (10) and (11). On the other hand, $E_{p}^{\max }$ from numerical simulations of ref[16] and [21] is much higher (see Fig.4).

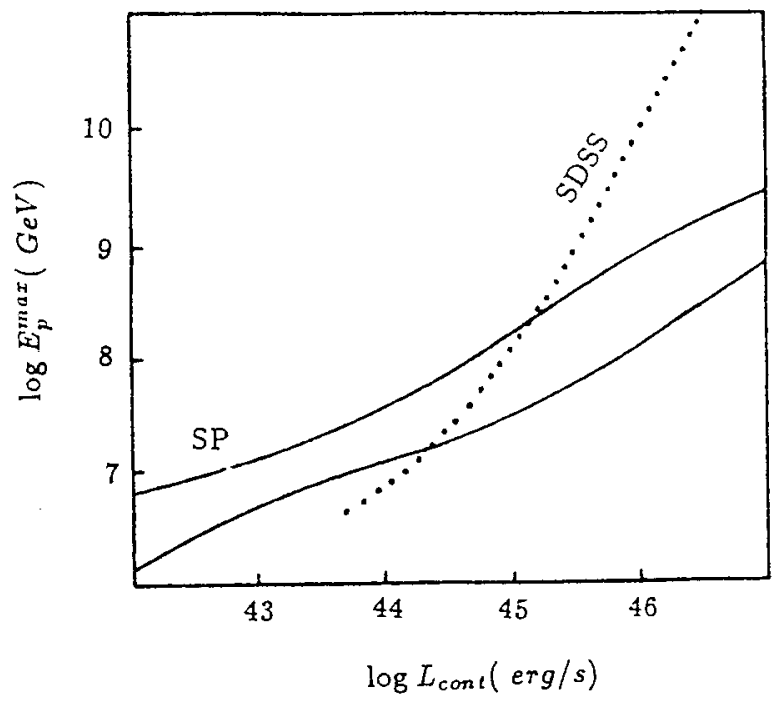

Fig.4. $E_{p}^{\max }$ as function of luminosity. SDSS curve corresponds to the calculations of ref[21] and SP - to ref[16]. We used here $\tilde{D}=3.3$.

A short comment is in order before we shall analyze the reason of this controversy.
From Fig. 3 one can see that the Eddington regime provides the lowest $E_{p}^{\max }$. This is due to large energy losses of protons. As luminosity decreases and mass of black hole increases, $E_{p}^{\text {max }}$ becomes larger. These trends are in accordance with Eq.(11).

Very large values of $E_{\bar{p}}^{\max }$ in the calculations $[11,16,17,21]$ are actually due to an additional assumption $L=k M$ with $\mathrm{k}$ being universal constant for the non-Eddington regimes. In this case $E_{p}^{\max }$ increases with $L$, as one can see it from Eq.(11).

As the most general case, luminosity is connected not with $M$ but with $\dot{M}$ according to the relation $L=\epsilon \dot{M} c^{2}$, where $\epsilon$ is the efficiency of the mass-energy conversion $(\epsilon \approx 0.1$ for spherical accretion and it can reach $\epsilon \approx 0.4$ for the rotating black hole). In case of quasi-spherical accretion $\dot{M}$ is mainly determined by the gas supply, i.e. it depends on the surroundings of the black hole but not necessarily on the black hole mass. For example, $\dot{M}$ can be small for rery massive black hole if it is submerged in the gas-poor elliptical galaxy: while it could be large for a black hole with moderate $M$ located in the dense gas cloud or in the star cluster. The disk accretion rate also depends on the gas supply to the disk. The Eddington regime with its rigorous mass-luminosity relation $L \propto M$ is the unique regime. The mass supply is assumed unlimited and the accretion rate is regulated by the radiation pressure. However, as it was discussed above, AGN in the Eddington regime are not efficient high-energy neutrino sources.

Therefore, it seems plausible to consider $E_{p}^{\max }$ as function of two parameters, $\dot{M}$ and $M$ (or $L$ and $M)$ as it is implied by Eq.(11). The AGN models for neutrino sources with two parameters $\dot{M}$ and $M$ (or $L$ and $M$ ) and with the condition $L<L_{E d d}$ (or $L \ll L_{E d d}$ ) seem to be more appropriate than the models with the rigid universal condition $L=$ $k M$.

The rigid relation $L=k M$ looks also unsatisfactory from the point of view of cosmological evolution, which implies that luminosity increases with redshift $z$. The $L=k M$ relation suggests that the black hole masses increase with $z$ too, 
while the accretion implies on contrary that the masses increase with time.

A reasonable assumption can be that while the masses of AGN, the powerful neutrino sources, are confined within the narrow range $10^{8}$ $10^{9} \mathrm{H}_{\mathrm{S}}$, their neutrino luminosities are varied within the wide range $10^{42}-10^{45} \mathrm{erg} / \mathrm{s}$.

\section{Conclusions}

The analysis presented here suggests that the maximum neutrino energy in the diffuse spectrum is limited by $10^{5}-10^{6} \mathrm{GeV}$. The maximum energy increases with the mass of a black hole $M$ and decreases as neutrino luminosity $L_{\nu}$ becomes larger. Neutrinos with the highest energies $\left(E_{\nu} \sim E_{\nu}^{\max }\right)$ in the diffuse spectrum are produced by the present-day $(z \approx 0)$ sources with small $L_{\nu}$ and large $M$, while strongly evolving sources (with large $L_{\nu}$ ) enhance the low-energy part of the spectrum produced at large redshift. We argue that maximum neutrino energy in the non-Eddington regime is function of two parameters $\dot{M}$ and $M$ (or $L$ and $M$ ). A plausible assumption could be that black hole masses $M$ for AGN - neutrino sources are confined within the narrow mass interval $10^{8}-10^{9} M_{\odot}$, while the luminosities are spread over much wider limits.

\section{REFERENCES}

1. V.Berezinsky and L.M.Ozernoy, Astron.Astroph.,98(1981)50.

2. C.T.Hill and D.N.Schramm, Phys. Rev.D $31(1985) 564$
3. E.V.Bugaev and E.A. Osipova, Moscow Univ. preprint 124 (1989).

4. J.Ellis et al, Nucl.Phys.B 373(1992) 399.

5. V.Berezinsky, Nucl.Phys.B $380(1992) 478$.

6. V.Berezinsky, A.Masiero and J.W.F.Valle, Phys.Lett.B 226(1991)382 .

7. V.Berezinsky, in Proc. Neutrino 77 Conf.(1977)177.

8. V.Berezinsky, S.Bulanov, V.Dogiel, V.Ginzburg and V.Ptuskin, Astrophysics of Cosmic Rays, North Holland (1990).

9. R.Silberberg and M.Shapiro, in Proc. 16th Int. Cosmic Ray Conf. (Kyoto), 10(1979)357.

10. T.Stanev, Nucl.Phys.(Proc.Suppl.) $35(1994) 185$.

11. F.W.Stecker, C.Done: MI.H.Salamon and P.Sommers, Phys.Rev.Lett 66(1991)2697.

12. A.Mastichiadis and J.G.Kirk, in [13], p.63.

13. V.J.Stenger, J.G.Learned, S.Pakvasa and X.Tata (eds), Proc. Workshop High Energy Neutrino Astronomy, World Scientific (1992).

14. V.Berezinsky, Nucl.Phys.(Proc.Suppl.) $28 \mathrm{~A}(1992) 352$.

15. F.W.Stecker, C.Done, M.H.Salamon and P.Sommers (Erratum) Phys.Rev.Lett 69(1992)2738.

16. A.P.Szabo and R.J.Protheroe, in [13], p. 24.

17. A.P.Szabo and R.J.Protheroe, Nucl.Phys.(Proc.Suppl.) 35(1994)287.

18. P.L.Biermann, in $[13\}, p .86$.

19. M.Sikora and M.C.Begelman, in [13], p.114.

20. K.Mannheim, Astron.Astroph. 269(1993)67.

21. F.W.Stecker, C.Done, M.H.Salamon and P.Sornmers, in [13], p.1. 